\title{
OUTLET POINTS AND HOMOGENEOUS CONTINUA
}

\author{
PAWEL KRUPSKI AND JANUSZ R. PRAJS
}

\begin{abstract}
A proof is presented for Bing's conjecture that homogeneous, treelike continua are hereditarily indecomposable. As a consequence, each homogeneous curve admits the continuous decomposition into the maximal terminal, homeomorphic, homogeneous, hereditarily indecomposable, treelike subcontinua. (2) A homogeneous, hereditarily unicoherent continuum contains either an arc or arbitrarily small, nondegenerate, indecomposable subcontinua. (3) A treelike continuum with property $K$ which is homogeneous with respect to confluent light mappings contains no two nondegenerate subcontinua with the one-point intersection.
\end{abstract}

\section{INTRODUCTION}

In 1960 Bing [2, p. 210] posed a conjecture that homogeneous, treelike continua are hereditarily indecomposable. It is a part of a more general and still open problem whether the pseudoarc is the only nondegenerate, homogeneous, treelike continuum. The Bing conjecture appeared to be of considerable interest and there are some partial solutions (see $[6-10,13])$.

In [13] the notion of an outlet point was introduced and it was proved that a nondegenerate, homogeneous, treelike continuum contains arbitrarily small subcontinua with at least two distinct outlet points. The second author, inspired by the result, has obtained a solution of Bing's conjecture in the affirmative (announced in [19]).

This paper provides a full and self-contained proof that homogeneous, treelike continua are hereditarily indecomposable (Theorem 6.4). It follows that each homogeneous curve $X$ admits continuous decomposition into the maximal terminal, homeomorphic, homogeneous, hereditarily indecomposable, treelike subcontinua (Theorem 6.6). In the case where $X$ is decomposable the decomposition coincides with the known Jones aposyndetic decomposition [24, Theorem 5 , p. 377]. If $X$ is indecomposable, then Theorem 6.6 gives a partial answer to Question 6 in $[25$, p. 221]. In the case of atriodic $X$ we obtain Hagopian's classification of all atriodic, homogeneous continua [8, Theorem 6.9].

Received by the editors October 30, 1987 and, in revised form, April 12, 1988.

1980 Mathematics Subject Classification (1985 Revision). Primary 54F20, 54F50, 54F55, 54C10.

Key words and phrases. Homogeneous continuum, treelike continuum, indecomposable continuum, terminal continuum, outlet point, Effros theorem, property $K$, confluent light mapping. 
We would also like to indicate a few other theorems of this paper which not only are steps in proving the main result but also seem to be interesting in their own right.

In $\S 3$ we consider treelike continua $X$ with the property of Kelley and we show

(F) $\quad X$ contains a sequence of nondegenerate subcontinua $Y_{k}, k=$ $1,2, \ldots$, converging to a point such that each $Y_{k}$ has an outlet point and $\bigcap_{k=1}^{\infty} Y_{k} \neq \varnothing$

(Theorem 3.4). It turns out that property $(\mathscr{F})$, together with Theorem 5.2, plays a fundamental role in $\S \S 4-6$.

Another consequence of Theorem 3.4 is Corollary 4.4: no treelike continuum with the property of Kelley which is homogeneous with respect to confluent light mappings contains two nondegenerate subcontinua with the one-point intersection.

In $\S 5$ we investigate a structure of homogeneous, hereditarily unicoherent continua. In particular, Theorem 5.2 says that a nondegenerate, homogeneous, hereditarily unicoherent continuum contains either an arc or arbitrarily small, nondegenerate, indecomposable subcontinua.

Key notions of the paper are outlet point and a terminal continuum; a main tool is the Effros theorem. Furthermore, an elementary covering techniquesome combinatorial properties of tree-chain covers-appears in $\S 3$.

\section{PRELiminaries}

Throughout the paper all spaces are metric and all mappings are continuous. Definitions of all notions not recalled here can be found in [14], [15], or [18].

We denote by diam $X$ the diameter of a space $X . C(X)$ is the hyperspace of all nonempty subcontinua of a compact space $X$ with the Hausdorff distance, and Ls and Lim denote the upper limit and the topological limit, respectively, in the sense of $[14$, pp. 337, 339].

The following proposition is obvious.

Proposition 2.1. If $C_{n}, n=1,2, \ldots$, are indecomposable subcontinua of $a$ continuum $C \in C(X)$, where $X$ is a hereditarily unicoherent continuum and $\operatorname{Lim}_{n} C_{n}=C$, then $C$ is indecomposable. Moreover, if $p \in X$, then there is a maximal indecomposable subcontinuum of $X$ containing $p$.

The space $X^{X}$ of all mappings from a compact space $X$ into $X$ is equipped with the metric $\rho(f, g)=\sup \{d(f(x), g(x)): x \in X\}$, where $d$ is a metric on $X$. The group of all homeomorphisms of $X$ onto $X$ is denoted by $H(X)$. If $\rho\left(h, i d_{X}\right)<\varepsilon$ and $h \in H(X)$, then we say that $h$ is an $\varepsilon$-homeomorphism.

A space $X$ is said to be homogeneous with respect to a class $M \subset X^{X}$ if for every $x, y \in X$ there exists a surjection $f \in M$ such that $f(x)=y . X$ is homogeneous provided it is homogeneous with respect to $H(X)$. 
We will use intensively the Effros theorem [5, Theorem 2.1, p. 39] for homogeneous, compact spaces in the following form (see [29, (1), p. 397]).

Effros' Theorem 2.2. If $X$ is a compact, homogeneous space then for every $\varepsilon>0$ there exists $a \delta>0$ such that if $d(x, y)<\delta$ then there is an $\varepsilon$-homeomorphism $h \in H(X)$ satisfying $h(x)=y$.

The number $\delta$ is called Effros' $\delta$ for $\varepsilon$.

The next proposition is a simple observation.

Proposition 2.3. Let $A \subset X$. For every $\delta>0$ the following statements are equivalent:

(a) for each $\delta$-homeomorphism $h \in H(X), h(A) \cap A \neq \varnothing \Rightarrow h(A) \subset A$;

(b) for each $\delta$-homeomorphism $h \in H(X), h(A) \cap A \neq \varnothing \Rightarrow A \subset h(A)$;

(c) for each $\delta$-homeomorphism $h \in H(X), h(A) \cap A \neq \varnothing \Rightarrow A=h(A)$.

Denote by $K(X)$ the family of all nonempty subcontinua of $X$ such that there is a $\delta>0$ satisfying condition (a) above. The following fact is an easy consequence of Proposition 2.3 and Effros' Theorem 2.2. Actually, one can find a proof in [17, (6.9), p. 17].

Proposition 2.4. Let $X$ be a compact, homogeneous space and $A \in K(X)$. Then for all $x, y \in A$ there is a homeomorphism $h \in H(X)$ such that $h(x)=y$ and $h(A)=A$. In particular, $A$ is homogeneous.

If a point $a \in X$ is fixed, we define the evaluation mapping $T_{a}: X^{X} \rightarrow X$ by $T_{a}(f)=f(a)$.

Recall that the quasi-interior of $A \subset X$ is the set $A^{*}=\bigcup\{U: U$ is open in $X$ and $U-A$ is of the first category $\}$.

The following proposition, which is an analog of the Effros theorem [5, Theorem 2.1], was proved in [4, Proposition 3.1, p. 584].

Proposition 2.5. If a compact space $X$ is homogeneous with respect to a Borel set $M \subset X^{X}$ and $a \in X$, then there is a surjection $h \in M$ such that $h(a) \in T_{a}(H)^{*}$ for each $H$ open in $M$ with $h \in H$.

Let us recall that a mapping $f$ of a compact space $X$ onto $Y$ is said to be confluent if, for every $A \in C(Y)$ and for each component $B$ of $f^{-1}(A)$, we have $f(B)=A$.

It is known that open mappings between compact spaces are confluent [30, (7.5), p. 148].

A mapping $f: X \rightarrow Y$ is light provided $f^{-1}(y)$ is 0 -dimensional for every $y \in Y$.

The set of all confluent light mappings of a compact space $X$ onto $X$ forms a $G_{\delta}$-subset of $X^{X}$ (see, e.g., [4, p. 581]).

A point $a \in A \in C(X)$ is called an outlet point of $A$ if $a \in Z$ for every $Z \in C(X)$ such that $Z \cap A \neq \varnothing \neq Z-A$ [13].

The set of all outlet points of $A$ will be denoted by $F(A)$. 
Proposition 2.6. Let $Y, K, L$ be subcontinua of a continuum $X$ such that $Y=$ $K \cup L$. Then

(a) if $F(K) \cap L \neq \varnothing \neq K-L$ and $F(L) \neq \varnothing$, then $F(K) \cup F(L) \subset F(Y)$;

(b) if $F(K) \cap F(L) \neq \varnothing$, then $F(K) \cup F(L) \subset F(Y)$.

Proof. Let $Z \in C(X)$ and $Z \cap Y \neq \varnothing \neq Z-Y$. We have to show that $F(K) \cup F(L) \subset Z$.

(a) Assume $Z \cap K \neq \varnothing$. Then $F(K) \subset Z$, for obviously $Z-K \neq \varnothing$. Therefore $\varnothing \neq F(K) \cap L \subset Z$ and thus $Z \cap L \neq \varnothing$. This implies that $F(L) \subset$ $Z$. Assume $Z \cap L \neq \varnothing$. Then $F(L) \subset Z$. We also have $F(L) \subset K$, thus $Z \cap K \neq \varnothing$. This implies $F(K) \subset Z$.

(b) Assume $Z \cap K \neq \varnothing$. Then $F(K) \subset Z$ and thus $\varnothing \neq Z \cap F(L) \subset Z \cap L$. Hence $F(L) \subset Z$. The proof for the case $Z \cap L \neq \varnothing$ is similar.

Observe that $F(A)=\bigcap\{C \in C(X): C-A \neq \varnothing \neq C \cap A\}$. Hence, we have the following proposition.

Proposition 2.7. $F(A)$ is a closed subset of $A$ and if $X$ is a hereditarily unicoherent continuum, then $F(A) \in C(A)$.

The next statement follows easily from the definitions.

Proposition 2.8. If $f: X \rightarrow Y$ is a confluent mapping, then $f(F(A)) \subset F(f(A))$ for every $A \in C(X)$.

Let us recall that a continuum $X$ has the property of Kelley (property $K$ ) if for every $y \in X$, for every sequence $\left\{y_{n}\right\} \subset X$ converging to $y$, and for every $Y \in C(X)$ containing $y$ there exists a sequence $\left\{Y_{n}\right\} \subset C(X)$ converging to $Y$ such that $y_{n} \in Y_{n}, n=1,2, \ldots[12,3.2$, p. 26].

Continua which are homogeneous with respect to open mappings have property $K$ [3, Statement, p. 380] but homogeneity with respect to confluent mappings does not imply the property [11].

A continuum $T \subset X$ is said to be terminal provided that if $C \in C(X)$ and $C \cap T \neq \varnothing$, then $C \subset T$ or $T \subset C$. Equivalently, $T$ is terminal if and only if $T=F(T)$.

Denote by $\mathscr{T}_{X}$ the family of all terinal subcontinua of a continuum $X$.

The following fact is well known and easy.

Proposition 2.9. If $X$ has property $K$, then $\mathscr{T}_{X}$ is closed in $C(X)$.

A simple proof of the next proposition is left to the reader.

Proposition 2.10. If a continuum $X$ has property $K, A_{k} \in C(X), k=1,2, \ldots$, and $\operatorname{Lim}_{k} A_{k}=A$, then $\operatorname{Ls}_{k} F\left(A_{k}\right) \subset F(A)$.

We will study properties of the following class $\mathscr{F}$ of continua. A continuum $X \in \mathscr{F}$ if and only if there is a sequence $\left\{Y_{k}\right\} \subset C(X), k=1,2, \ldots$, such that $Y_{k}$ is nondegenerate, $F\left(Y_{k}\right) \neq \varnothing, \lim _{k} \operatorname{diam} Y_{k}=0$, and $\bigcap_{k} Y_{k} \neq \varnothing$.

Finally, let us recall terminology connected with treelikeness. A finite colleciton $\mathscr{C}$ of open subsets of $X$ is called a tree-chain if its nerve is a tree. 
Elements of $\mathscr{C}$ are called links. Let $C \in \mathscr{C}$. If $\mathscr{C}-\{C\}$ is again a tree-chain, then $C$ is said to be an end-link of $\mathscr{C}$. Otherwise, $C$ is called a separating link of $\mathscr{C}$ and maximal tree-chains in $\mathscr{C}-\{C\}$ are called branches of $\mathscr{C}$ (associated with $C$ ).

A continuum $X$ is treelike if there exists a sequence of tree-chains $\mathscr{C}_{n}$, $n=1,2, \ldots$, covering $X$ such that $\mathscr{C}_{n+1}$ refines $\mathscr{C}_{n}$ and $\lim _{n} \operatorname{mesh} \mathscr{C}_{n}=0$. The sequence $\left\{\mathscr{C}_{n}\right\}$ will be called a defining sequence of $X$.

\section{OUTLET POINTS IN TREELIKE CONTINUA WITH PROPERTY $K$}

In this section we show that each nondegenerate treelike continuum with property $K$ belongs to the class $\mathscr{F}$ (Theorem 3.4). Lemma 3.2 and Theorem 3.4 appeared in [13] in a weaker form; we modify their proofs here.

Lemma 3.1. Let $\alpha$ be a positive number and let $\mathscr{D}$ be a tree-chain in a space $X$ such that mesh $\mathscr{D}<\alpha / 3<\alpha<$ diam $\cup \mathscr{D}$. Denote by $\mathscr{D}^{0}$ the family of all branches of $\mathscr{D}$ having diameters $<\alpha / 3$ and by $\mathscr{D}^{1}$ the set of all maximal (with respect to the inclusion) elements of $\mathscr{D}^{0}$. Let $\mathscr{C}^{0}$ be the set of all $C \in \mathscr{D}$ such that there exists a branch in $\mathscr{D}^{1}$ associated with $C$. Fix an end-link $D$ of $\mathscr{D}$. Then there is $C \in \mathscr{C}^{0}$ such that

(a) a branch in $\mathscr{D}^{1}$ associated with $C$ does not contain $D$;

(b) exactly one branch $\mathscr{B}$ associated with $C$ has diameter $\geq \alpha / 3$; and

(c) $D \in \mathscr{B}$.

Proof. Suppose the contrary. Take a branch in $\mathscr{D}^{1}$ not containing $D$, associated with some $C_{1} \in \mathscr{C}^{0}$ (clearly, it exists). Then there is a branch $\mathscr{B}_{1}$ associated with $C_{1}$ of diameter $\geq \alpha / 3$ such that $D \notin \mathscr{B}_{1}$. Again, $\mathscr{B}_{1}$ contains a branch from $\mathscr{D}^{1}$ associated with some $C_{2} \in \mathscr{C}^{0} \cap \mathscr{B}_{1}$. Consequently, there is a branch $\mathscr{B}_{2} \varsubsetneqq \mathscr{B}_{1}$ associated with $C_{2}$ of diameter $\geq \alpha / 3$ such that $D \notin \mathscr{B}_{2}$, etc. Continuing this process we get a contradiction, since the tree-chain $\mathscr{D}$ is finite.

In the remaining part of this chapter $X$ will denote a nondegenerate treelike continuum with defining sequence $\left\{\mathscr{C}_{n}\right\}$.

A subcontinuum $Y$ of $X$ is said to be a limit of branches if there are numbers $n_{1}<n_{2}<\cdots$ and branches $\mathscr{D}_{n_{k}}$ of $\mathscr{C}_{n_{k}}, k=1,2, \ldots$, such that $Y=$ $\operatorname{Lim}_{k} \cup \mathscr{D}_{n_{k}}$.

Lemma 3.2. There is a sequence $Y_{k}, k=1,2, \ldots$, of nondegenerate subcontinua of $X$ such that for each $k$

(i) $Y_{k}$ is a limit of branches,

(ii) $Y_{k+1} \subset Y_{k}$,

(iii) $\bigcap_{k=1}^{\infty} Y_{k}$ is degenerate.

Proof. It is clear that $X$ contains a nondegenerate limit of branches $Y_{1}$. Suppose $Y_{1}, Y_{2}, \ldots, Y_{k-1}=Y$ satisfy (i) and (ii). Let $0<\alpha<\operatorname{diam} Y / 2$. It suffices to construct a nondegenerate limit of branches $Y_{k} \subset Y$ with diam $Y_{k} \leq \alpha$. 
We can assume $Y=\operatorname{Lim}_{n} \cup \mathscr{D}_{n}$, where $\mathscr{D}_{n}$ is a branch of $\mathscr{C}_{n}$ associated with a link $E_{n} \in \mathscr{C}_{n}$, and $\alpha<\operatorname{diam} \bigcup \mathscr{D}_{n}, \operatorname{mesh} \mathscr{D}_{n}<\alpha / 3, n=1,2, \ldots$ Let $D_{n}$ be an end-link of $\mathscr{D}_{n}$ not intersecting $E_{n}$. For each $n$ there exist a link $C_{n} \in \mathscr{D}_{n}$ and a branch $\mathscr{B}_{n}$ of $\mathscr{D}_{n}$ satisfying conditions (a)-(c) in Lemma 3.1 for $D=D_{n}$ and $\mathscr{D}=\mathscr{D}_{n}$. Then a link $B_{n} \in \mathscr{B}_{n}$ intersects $C_{n}$. Further, the branch $\mathscr{A}_{n}$ associated with $B_{n}$ containing all branches of $\mathscr{D}_{n}$ associated with $C_{n}$ which are different from $\mathscr{B}_{n}$ is also a branch of $\mathscr{C}_{n}$ associated with $B_{n}$ and $\operatorname{diam} \bigcup \mathscr{A}_{n}<\alpha$. We can assume that the sequence $\left\{\bigcup \mathscr{A}_{n}\right\}, n=1,2, \ldots$, is convergent; put $\operatorname{Lim}_{n} \cup \mathscr{A}_{n}=Y_{k}$. Observe that $Y_{k} \in C(X)$ and $\operatorname{diam} Y_{k} \leq \alpha$. Moreover, $Y_{k}$ is nondegenerate, because otherwise diam $\bigcup \mathscr{A}_{n}<\alpha / 3$ for sufficiently large $n$; this is impossible because $\mathscr{A}_{n}$ properly contains a branch from $\left(\mathscr{D}_{n}\right)^{1}$ associated with $C_{n}$. So $Y_{k}$ is the desired limit of branches and the proof is complete.

Lemma 3.3. If $X$ has property $K$ and a subcontinuum $Y$ of $X$ is a limit of branches, then $Y$ contains an outlet point.

Proof. Let $Y=\operatorname{Lim}_{n} \cup \mathscr{D}_{n}$, where $\mathscr{D}_{n}$ is a branch of $\mathscr{C}_{n}$ associated with a link $C_{n}$ of $\mathscr{C}_{n}$. Assume $\operatorname{Lim}_{n} C_{n}=\{p\} \subset Y$. We will show that $p$ is an outlet point of $Y$. Indeed, suppose $Z \in C(X)$ and $Z \cap Y \neq \varnothing \neq Z-Y$. Take $y \in Z \cap Y$ and a sequence of points $y_{n} \in \bigcup \mathscr{D}_{n}, n=1,2, \ldots$, converging to $y$. By property $K$, there exists a sequence $\left\{Z_{n}\right\} \subset C(X), n=1,2, \ldots$, converging to $Z$ such that $y_{n} \in Z_{n}$. Since $Z-Y \neq \varnothing$, we also have $Z_{n}-\bigcup \mathscr{D}_{n} \neq \varnothing$ for sufficiently large $n$, whence $Z_{n} \cap C_{n} \neq \varnothing$. So $p \in Z$.

The following theorem is a consequence of Lemmas 3.2 and 3.3.

Theorem 3.4. If a treelike continuum $X$ has property $K$, then $X$ contains $a$ sequence of nondegenerate subcontinua $Y_{k}, k=1,2, \ldots$, such that for each

$k$ (i) $Y_{k}$ has an outlet point,

(ii) $Y_{k+1} \subset Y_{k}$,

(iii) $\bigcap_{k=1}^{\infty} Y_{k}$ is degenerate.

In particular, $X \in \mathscr{F}$.

\section{CONTINUA With PROPERTy $K$ WHich ARE HOMOgENEOUS WITH RESPECT TO CONFLUENT LIGHT MAPPINGS}

In this section we will study continua from the class $\mathscr{F}$ with property $K$ which are homogeneous with respect to confluent light mappings. The class $\mathscr{F}$ is naturally divided into the following two classes: the class $\mathscr{F}_{0}$ of all continua $X$ containing a sequence $\left\{Y_{k}\right\}$ of nondegenerate subcontinua such that $\operatorname{Lim}_{k} Y_{k}=\bigcap_{k=1}^{\infty} Y_{k}=\{p\}$ for some $p \in X$ and $p \in F\left(Y_{k}\right)$ for each $k$, and the class $\mathscr{F}_{1}=\mathscr{F}-\mathscr{F}_{0}$.

Lemma 4.1. Let a continuum $X \in \mathscr{F}_{0}$ have property $K$ and be homogeneous with respect to confluent light mappings. Then for each $x \in X$ there is a sequence 
$\left\{Z_{k}\right\} \subset C(X), k=1,2, \ldots$, such that $\operatorname{Lim}_{k} Z_{k}=\bigcap_{k=1}^{\infty} Z_{k}=\{x\}$ and $F\left(Z_{k}\right)$ contains nondegenerate subcontinua for each $k$.

Proof. Let a sequence $\left\{Y_{k}\right\} \subset C(X), k=1,2, \ldots$, be guaranteed by the definition of $\mathscr{F}_{0}$. Further, let $h: X \rightarrow X$ be a light confluent surjection described in Proposition 2.5 for the class $M$ of all confluent light mappings and for $\{a\}=\operatorname{Lim}_{k} Y_{k}$. Note that since $h$ is light, each $h\left(Y_{k}\right)$ is nondegenerate. By Proposition 2.8, $h(a)$ is an outlet point of $h\left(Y_{k}\right)$ for each $k$ and, evidently, $\{h(a)\}=\bigcap_{k=1}^{\infty} h\left(Y_{k}\right)$. Fix a positive integer $k$. Let $m$ be such that $\operatorname{diam} h\left(Y_{m}\right)<1 / 4 k$ and take a positive number $\varepsilon<\operatorname{diam} h\left(Y_{m}\right) / 2$. There exists a $\delta>0$ such that $B(h(a), \delta) \subset T_{a}(H)^{*}$, where $B(h(a), \delta)$ denotes the $\delta$-ball about $h$ in $M$. Let $n$ be such that $n>m, h\left(Y_{n}\right) \subset B(h(a), \delta)$, and $\operatorname{diam} h\left(Y_{n}\right)<\operatorname{diam} h\left(Y_{m}\right)-2 \varepsilon$.

Choose a dense countable subset $\left\{q_{1}, q_{2}, \ldots\right\}$ of $h\left(Y_{n}\right)$. For each positive integer $i$ we construct a continuum $K_{i}$ with $h(a), q_{i} \in F\left(K_{i}\right)$ in the following way. Since $q_{i} \in T_{a}(H)^{*}$ and $T_{a}(H)$ is dense in $T_{a}(H)^{*}$, there exists a sequence of points $p_{s} \in T_{a}(H), s=1,2, \ldots$, converging to $q_{i}$. Hence, for each $s$ there is $h_{s} \in H$ carrying $a$ onto $p_{s}$. Take a convergent subsequence of subcontinua $h_{s_{j}}\left(Y_{m}\right), j=1,2, \ldots$, and denote its limit by $L_{i}$. In view of Proposition 2.8, $p_{s_{j}}$ is an outlet point of $h_{s_{j}}\left(Y_{m}\right)$ and, by Proposition 2.10, $q_{i}$ is an outlet point of $L_{i}$. Note that $\operatorname{diam} h\left(Y_{n}\right)<\operatorname{diam} L_{i}$. Define $K_{i}=h\left(Y_{n}\right) \cup L_{i}$.

Observe that $\operatorname{diam} K_{i} \leq 2 \operatorname{diam} h\left(Y_{m}\right)<1 / 2 k$. Since $h(a) \in F\left(h\left(Y_{n}\right)\right)$, $q_{i} \in F\left(L_{i}\right) \cap h\left(Y_{n}\right)$, and $L_{i}-h\left(Y_{n}\right) \neq \varnothing$, we conclude that $h(\alpha), q_{i} \in F\left(K_{i}\right)$ by Proposition 2.6(a). Put $P_{i}=K_{1} \cup \cdots \cup K_{i}$ and $Z_{k}=\operatorname{Lim}_{i} P_{i}$. We have $\left\{q_{1}, \ldots, q_{i}\right\} \subset F\left(P_{i}\right)$ by Proposition 2.6(b) and $\operatorname{cl}\left\{q_{1}, q_{2}, \ldots\right\}=h\left(Y_{n}\right) \subset$ $F\left(Z_{k}\right)$ by Proposition 2.10. Observing that $\operatorname{diam} Z_{k} \leq 1 / k$ we see that the desired sequence is already constructed for $x=h(a)$. To complete the proof note that for any confluent light mapping $f: X \rightarrow X$ the sequence $\left\{f\left(Z_{k}\right)\right\}$ has the same properties as $\left\{Z_{k}\right\}$, hence the conclusion follows from the homogeneity of $X$ with respect to confluent light mappings.

The following theorem was obtained in [13] in a version for homogeneous treelike continua.

Theorem 4.2. If a nondegenerate continuum $X \in \mathscr{F}$ has property $K$ and $X$ is homogeneous with respect to confluent light mappings, then there exists a sequence $\left\{Z_{k}\right\} \subset C(X), k=1,2, \ldots$, such that, for each $k, F\left(Z_{k}\right)$ is a nondegenerate subset of $Z_{k}, \operatorname{Lim}_{k} Z_{k}=\bigcap_{k=1}^{\infty} Z_{k}=\{p\}$ for some $p \in X$.

Proof. If $X$ is in $\mathscr{F}_{0}$, the conclusion follows from Lemma 4.1. Assume $X \in$ $\mathscr{F}_{1}$. Let $\left\{Y_{k}\right\}, k=1,2, \ldots$, be a sequence of subcontinua of $X$ with outlet points $p_{k} \in Y_{k}, \bigcap_{k=1}^{\infty} Y_{k}=\{p\}, p_{k} \neq p$ for each $k$. Fix a positive integer $k$. Take $Y_{n}$ with diam $Y_{n}<1 / 2 k$. There exists a confluent light mapping $f$ of $X$ onto $X$ such that $f(p)=p_{n}$. Subcontinua $f\left(Y_{k}\right), k=1,2, \ldots$, again form a sequence such that $F\left(f\left(Y_{k}\right)\right) \neq \varnothing$ and $\bigcap_{k=1}^{\infty} f\left(Y_{k}\right)=\left\{p_{n}\right\}$. Since $X \in \mathscr{F}_{1}$, we can assume that each $f\left(Y_{k}\right)$ has an outlet point $q_{k} \neq p_{n}$. Let $m$ be such that $\operatorname{diam} f\left(Y_{m}\right)<\operatorname{diam} Y_{n}$. Put $Z_{k}=Y_{n} \cup f\left(Y_{m}\right)$. Since $p_{n} \in F\left(Y_{n}\right) \cap f\left(Y_{m}\right)$, 
$q_{m} \in F\left(f\left(Y_{m}\right)\right)$, and $Y_{n}-f\left(Y_{m}\right) \neq \varnothing$, in view of Proposition 2.6(a), the set $F\left(Z_{k}\right)$ is nondegenerate. We also have $\operatorname{diam} Z_{k}<1 / k$ and $p \in Z_{k}$ for each $k$. So the proof is complete.

Theorem 4.3. Let $X \in \mathscr{F}$ with property $K$ be homogeneous with respect to confluent light mappings. Then for nondegenerate continua $K, L \in C(X)$ the intersection $K \cap L$ has no isolated point. In particular, $X$ contains no two nondegenerate subcontinua with one-point intersection, and thus $X$ contains no arc.

Proof. Let $x \in K \cap L$, where $K, L \in C(X)$ are nondegenerate. Assume $X \in$ $\mathscr{F}_{0}$. Let $\left\{Z_{k}\right\}$ be a sequence guaranteed by Lemma 4.1 with $\bigcap_{k=1}^{\infty} Z_{k}=\{x\}$. Then $F\left(Z_{k}\right) \subset K \cap L$ for sufficiently large $k$, and thus the conclusion follows.

Assume $X \in \mathscr{F}_{1}=\mathscr{F}-\mathscr{F}_{0}$. Then there is a sequence $\left\{Y_{k}\right\}$ of nondegenerate subcontinua of $X$ satisfying $F\left(Y_{k}\right) \neq \varnothing$ and $\operatorname{Lim}_{k} Y_{k}=\bigcap_{k=1}^{\infty} Y_{k}=\{p\}$ for some $p \in X$. By Proposition 2.8 and by the homogeneity of $X$ with respect to confluent light mappings, there is such a sequence for each $p \in X$. So assume $p=x$. Since $X \in \mathscr{F}_{1}$, there is $p_{k} \in F\left(Y_{k}\right)-\{x\}$ for almost all $k$, therefore $p_{k} \in K \cap L$ for such $k$. The proof is thus complete.

The following corollary is a simple consequence of Theorems 3.4 and 4.3.

Corollary 4.4. Each treelike continuum with property $K$ which is homogeneous with respect to confluent light mappings contains no two nondegenerate subcontinua with the one-point intersection.

The next corollary is a generalization of [7, Comments, p. 563] (see also [13, Corollary 3.4]).

Corollary 4.5. Each treelike continuum which is homogeneous with respect to open light mappings contains no two nondegenerate subcontinua with the onepoint intersection.

\section{HOMOGENEOUS, HEREDITARILY UNICOHERENT CONTINUA}

Let us recall an old result by Jones [9].

Proposition 5.1. A homogeneous, hereditarily unicoherent continuum is indecomposable.

The following theorem is crucial for further investigations.

Theorem 5.2. Each nondegenerate, homogeneous, hereditarily unicoherent continuum $X$ contains either an arc or arbitrarily small, nondegenerate, indecomposable subcontinua.

Proof. Suppose, on the contrary, that $X$ contains no arc and there is a $\delta>0$ such that each nondegenerate subcontinuum of $X$ of diameter less than $\delta$ is decomposable. 
If $I \subset X$ is a nondegenerate, irreducible continuum with $\operatorname{diam} I<\delta$, then there is a finest monotone map $m$ of $I$ onto $[0,1]$ (see $[15$, Theorem $3, \mathrm{p}$. 200]).

We begin with the following fact.

Claim 1. There is an irreducible continuum $I \subset X$ with a finest monotone map $m: I \rightarrow[0,1]$ such that

(1) $\operatorname{diam} I<\delta / 2$ and the continua $m^{-1}(0), m^{-1}(1)$ are nondegenerate.

Indeed, let $I_{0}$ be an arbitrary irreducible subcontinuum of $X$ of diameter less than $\delta / 4$ with a finest monotone map $m: I_{0} \rightarrow m\left(I_{0}\right)=[0,1]$. Define $L(t)=m^{-1}(t) \cap \operatorname{cl}\left(m^{-1}([0, t))\right)$ and $R(t)=m^{-1}(t) \cap \operatorname{cl}\left(m^{-1}((t, 1])\right)$ for $t \in$ $(0,1)$. If there are $t_{1}, t_{2} \in(0,1), t_{1}<t_{2}$, with nondegenerate $R\left(t_{1}\right)$ and $L\left(t_{2}\right)$, then $\operatorname{cl}\left(m^{-1}\left(t_{1}, t_{2}\right)\right)$ can be the required continuum. Assume there are no $t_{1}, t_{2}$ as above and put $P=\{t \in(0,1): L(t)$ is nondegenerate $\}, Q=\{t \in$ $(0,1): R(t)$ is nondegenerate $\}, S=\left\{t \in(0,1): m^{-1}(t)\right.$ is nondegenerate $\}$. Since all layers $m^{-1}(t)$ are boundary sets (see [15, Theorem 5, p. 217]), $m^{-1}(t)$ $=L(t) \cup R(t)$ for $t \in(0,1)$. Hence $S=P \cup Q$ and $\operatorname{cl} S=[0,1]$, because $X$ contains no arc. Moreover, there is $t_{0} \in[0,1]$ such that $P \subset\left[0, t_{0}\right]$ and $Q \subset\left[t_{0}, 1\right]$. Assume $t_{0} \neq 0$ (the proof for the case $t_{0} \neq 1$ is similar). Fix two points $u, u_{1} \in P$ with $0<u<u_{1}<t_{0}$. Then $R(u)$ consists of a single point $p,\{p\} \varsubsetneqq L(u)$ and there is a sequence $\left\{p_{n}\right\} \subset m^{-1}([0, u))$ converging to $p$. By Effros' Theorem 2.2, there are $\varepsilon_{n}$-homeomorphisms $h_{n} \in H(X)$ such that $h_{n}(p)=p_{n}$ and $\lim _{n} \varepsilon_{n}=0$. Putting $I_{1}=\operatorname{cl}\left(m^{-1}\left(\left(u, u_{1}\right]\right)\right)$, we see that, for great $n, h_{n}\left(I_{1}\right)$ cannot contain $m^{-1}(u)$, thus $h_{n}\left(I_{1}\right) \cap I_{1}=\varnothing$ (otherwise $I_{0}$ is not irreducible). Hence, for sufficiently great $n$, the irreducible continuum $I \subset X$ intersecting $m^{-1}\left(u_{1}\right)$ and $h_{n}\left(m^{-1}\left(u_{1}\right)\right)$, contained in the union $h_{n}\left(I_{1}\right) \cup m^{-1}\left(\left[m\left(p_{n}\right), u\right]\right) \cup I_{1}$, can be the required continuum. In fact, for each finest monotone map $f: I \rightarrow[0,1]$, the layers $m^{-1}\left(u_{1}\right)$ and $h_{n}\left(m^{-1}\left(u_{1}\right)\right)$ are the end layers; that is, either $f^{-1}(0)=m^{-1}\left(u_{1}\right), f^{-1}(1)=h_{n}\left(m^{-1}\left(u_{1}\right)\right)$ or $f^{-1}(0)=h_{n}\left(m^{-1}\left(u_{1}\right)\right), f^{-1}(1)=m^{-1}\left(u_{1}\right)$.

Claim 2. If an irreducible continuum $I \subset X$ and a finest monotone map $m: I \rightarrow[0,1]$ satisfy $(1)$, then for every $\varepsilon>0$ there is an $\varepsilon$-homeomorphism $h \in H(X)$ such that

$$
\begin{gathered}
h\left(m^{-1}(1)\right) \cap m^{-1}(0)=\varnothing, \\
m^{-1}(0) \cap h\left(m^{-1}(0)\right) \neq \varnothing \neq m^{-1}(0)-h\left(m^{-1}(0)\right), \quad \text { and } \\
h(I) \cap I \subset m^{-1}(0) .
\end{gathered}
$$

In fact, since $m^{-1}(0)$ is decomposable, it is not in $K(X)$, by Propositions 5.1 and 2.4. It follows from Proposition 2.3 that there is an $\varepsilon$-homeomorphism $h \in H(X)$ satisfying (2). Suppose (3) is not fulfilled and put

$$
t_{0}=\sup \left\{t \in[0,1]: m^{-1}(t) \cap h(I) \neq \varnothing\right\} .
$$


The hereditary unicoherence of $X$ yields that the decomposition of $I$ into the sets $m^{-1}(t) \cap h\left(m^{-1}(u)\right)$ for $t \in\left[0, t_{0}\right), u \in[0,1]$ and the sets $m^{-1}(t)$ for $t \in$ $\left[t_{0}, 1\right]$ is monotone and upper semicontinuous with an arc as the decomposition space. But the element $h\left(m^{-1} \cdot(0)\right) \cap m^{-1}(0)$ of this decomposition is properly contained in $m^{-1}(0)$, a contradiction, for $m$ is the finest monotone map.

Now we use Claims 1 and 2 to construct (inductively) a nondegenerate, indecomposable subcontinuum of diameter less than $\delta$, thus obtaining a contradiction. Take $I_{0}$ and $m_{0}$ as guaranteed by Claim 1 and a homeomorphism $h_{1} \in H(X)$ guaranteed by Claim 2 for $I=I_{0}, m=m_{0}$, and $\varepsilon=\delta / 8$. Thus, the continuum $I_{1}=I_{0} \cup h_{1}\left(I_{0}\right)$ is irreducible between each pair of points $p \in m_{0}^{-1}(1)$ and $q \in h_{1}\left(m_{0}^{-1}(1)\right)$, and $m_{0}^{-1}(1), h_{1}\left(m_{0}^{-1}(1)\right)$ are the end layers of each finest monotone map $m_{1}: I_{1} \rightarrow[0,1]$. Fix such a map $m_{1}$ with $m_{1}^{-1}(0)=h_{1}\left(m_{0}^{-1}(1)\right)$. Let $h_{2} \in H(X)$ be a homeomorphism guaranteed by Claim 2 for $I=I_{1}, m=m_{1}$, and $\varepsilon=\delta / 16$. Put $I_{2}=I_{1} \cup h_{2}\left(I_{1}\right)$, etc.

In this way an increasing sequence $\left\{I_{n}\right\}$ of irreducible subcontinua of $X$ is obtained.

Claim 3. The continuum $K=\operatorname{Lim}_{n} I_{n}$ is indecomposable and $0<\operatorname{diam} K<$ $\delta$.

In fact, each $I_{n}$ is nondegenerate and $\operatorname{diam} I_{n+1} \leq \operatorname{diam} I_{n}+\delta / 2^{n+2}$, so $0<\operatorname{diam} K<\delta$. Suppose $K$ contains a subcontinuum $C$ with nonempty interior in $K$ and let $p \in \operatorname{int} C$. There is a sequence of points $p_{n} \in I_{n}-I_{n-1}$ converging to $p$. For sufficiently great $n$ we have $p_{n+1} \in C \cap\left(I_{n+1}-I_{n}\right)$ and $p_{n-1} \in C \cap\left(I_{n-1}-I_{n-2}\right)$. Since the set $I_{n}-I_{n-1}$ is contained in the irreducible subcontinuum of $X$ between $p_{n+1}$ and $p_{n-1}$, it follows from the hereditary unicoherence of $X$ that $I_{n}-I_{n-1} \subset C$. Noting that the sets $I_{n}-I_{n-1}, n=$ $1,2, \ldots$, approximate $K$, we see that $C=K$. Hence $K$ is indecomposable.

The proof of Theorem 5.2 is complete.

Proposition 5.3. Suppose $A$ is a proper subcontinuum of a homogeneous, hereditarily unicoherent continuum $X$. If $C$ is an indecomposable subcontinuum of $X$ intersecting $F(A)$, then either $F(A) \subset C$ or $C \subset F(A)$; if, additionally, $C \subset A$, then $C \subset F(A)$.

Proof. If $C-A \neq \varnothing$, then $F(A) \subset C$, by the definition of $F(A)$. Assume $C \subset A$ and suppose, on the contrary, that $C-F(A) \neq \varnothing$. Then there is a composant $C_{1}$ of $C$ contained in $C-F(A)$, by the hereditary unicoherence of $X$. Given $p \in C-F(A)$, we find $K \in C(X)$ satisfying $K \cap A \neq \varnothing \neq K-A$ and $p \notin K$. It follows from Effros' Theorem 2.2 that there is $h \in H(X)$ such that $h(K) \cap C_{1} \neq \varnothing$ and $h$ is so close to the identity map that $C-h(K) \neq$ $\varnothing \neq h(K)-A$. Then the continuum $h(K) \cap C$ lies in $C_{1}$ (here we use the hereditary unicoherence of $X)$, so $h(K)$ is disjoint with the only composant of $C$ intersecting $F(A)$, a contradiction. 
Proposition 5.4. Let $X$ be a homogeneous, hereditarily unicoherent continuum and $A \in C(X)$. Then each two maximal indecomposable subcontinua of $F(A)$ are either disjoint or equal (compare Proposition 2.1).

Proof. For each $x \in F(A)$ put $S_{x}=\{p \in F(A)$ : there are indecomposable subcontinua $C_{1}, \ldots, C_{n} \subset F(A)$ with $x \in C_{1}, p \in C_{n}$, and $C_{i} \cap C_{i+1} \neq \varnothing$ for $i=1, \ldots, n-1\}$. Observe that $x \in S_{x}$ and either $S_{x}=S_{y}$ or $S_{x} \cap S_{y}=\varnothing$.

Assume that for each $\delta>0$ there are a $\delta$-homeomorphism $h \in H(X)$ and a point $x \in F(A)$ satisfying $h\left(S_{x}\right) \cap F(A) \neq \varnothing \neq h\left(S_{x}\right)-F(A)$ and let $p \in h\left(S_{x}\right) S_{x} \cap F(A), q \in h\left(S_{x}\right)-F(A)$. Thus, there are indecomposable continua $C_{1}, \ldots, C_{n} \subset h\left(S_{x}\right)$ with $p \in C_{1}, q \in C_{n}$, and $C_{i} \cap C_{i+1} \neq \varnothing$, $i=1,2, \ldots, n-1$. In particular, there is $C_{k}$ satisfying $C_{k} \cap F(A) \neq \varnothing \neq$ $C_{k}-F(A)$. It follows from Proposition 5.3 that $F(A) \subset C_{k}$. Therefore the Hausdorff distance between the indecomposable continuum $h^{-1}\left(C_{k}\right) \subset F(A)$ and $F(A)$ is less than $\delta$. Since $\delta>0$ is arbitrary, $F(A)$ is indecomposable in view of Proposition 2.1. So the conclusion of Proposition 5.4 holds.

Assume now there is a $\delta>0$ such that for each $\delta$-homeomorphism $h \in$ $H(X)$ and for each $x \in F(A)$ if $h\left(S_{x}\right) \cap F(A) \neq \varnothing$, then $h\left(S_{x}\right) \subset F(A)$. Let $p \in \operatorname{cl} S_{x} \cap \operatorname{cl} S_{y}$. Thus, there are sequences $\left\{x_{n}\right\} \subset S_{x}$ and $\left\{y_{n}\right\} \subset S_{y}$ converging to $p$. Applying Effros' Theorem 2.2, note that for large $n$ there are $\varepsilon_{n}$-homeomorphisms $h_{n}, g_{n} \in H(X)$ such that $h_{n}(p)=x_{n}, g_{n}(p)=y_{n}$, $\varepsilon_{n}<\delta$, and $\lim _{n} \varepsilon_{n}=0$. Observe that $h_{n}\left(S_{p}\right) \subset S_{x}$ and $h_{n}^{-1}\left(S_{x}\right) \subset S_{p}$ by the definitions of $S_{x}$ and $S_{p}$. So $h_{n}\left(S_{p}\right)=S_{x}$ and, similarly, $g_{n}\left(S_{p}\right)=S_{y}$. It follows that $\operatorname{cl} S_{x}=\operatorname{Lim}_{n} h_{n}\left(\operatorname{cl} S_{p}\right)=\operatorname{Lim}_{n} g_{n}\left(\operatorname{cl} S_{p}\right)=\operatorname{cl} S_{y}$. Therefore the family $\left\{\operatorname{cl} S_{x}: x \in F(A)\right\}$ is a decomposition of $F(A)$. If $h \in H(X)$ is a $\delta$ homeomorphism and there is a point $p \in h\left(\operatorname{cl} S_{x}\right) \cap \mathrm{cl} S_{x}$, then $h\left(\operatorname{cl} S_{x}\right) \subset \operatorname{cl} S_{p}=$ $\operatorname{cl} S_{x}$. Hence, $\operatorname{cl} S_{x} \in K(X)$ for each $x \in F(A)$, so $\operatorname{cl} S_{x}$ is homogeneous (Proposition 2.4) and thus indecomposable (Proposition 5.1).

Finally, observe that each maximal indecomposable subcontinuum of $F(A)$ is of the form $\mathrm{cl} S_{x}$ by the definition of $S_{x}$. The proof of Proposition 5.4 is complete.

Denote by $\mathscr{D}_{X}$ the family of all indecomposable subcontinua $K$ of $X$ such that if $C \in C(X)$ is indecomposable and $C \cap K \neq \varnothing$, then either $C \subset K$ or $K \subset C$.

Proposition 5.5. Let $X$ be a homogeneous, hereditarily unicoherent continuum and $A \in C(X)$. Then each maximal indecomposable subcontinuum of $F(A)$ belongs to $\mathscr{D}_{X}$.

Proof. Let $K$ be a maximal indecomposable subcontinuum of $F(A)$ and let $C \in C(X)$ be indecomposable and $C \cap K \neq \varnothing$. Then, by Proposition 5.3, $C \subset F(A)$ or $F(A) \subset C$. If $C \subset F(A)$, then $C \subset K$ (Proposition 5.4); if $F(A) \subset C$, then $K \subset C$. 
Lemma 5.6. Let $X$ be a homogeneous continuum and let $C \in C(X)$ be indecomposable or $C \in \operatorname{cl}\left(\mathscr{D}_{X}\right)$. If $D \in \operatorname{cl}\left(\mathscr{D}_{X}\right)$ and $C \cap D \neq \varnothing$, then either $C \subset D$ or $D \subset C$.

Proof. Let $p \in C \cap D$ and a sequence $\left\{D_{n}\right\} \subset \mathscr{D}_{X}$ converge to $D$. In the case $C \in \operatorname{cl}\left(\mathscr{D}_{X}\right)$ take a sequence $\left\{C_{n}\right\} \subset \mathscr{D}_{X}$ converging to $C$. Without loss of generality, according to Effros' Theorem 2.2, we can assume $p \in D_{n}$ (and also $p \in C_{n}$ if $\left.C \in \operatorname{cl}\left(\mathscr{D}_{X}\right)\right)$ for all $n$. Thus, if $C$ is indecomposable, then $D_{n} \subset C$ for infinitely many $n$ or $C \subset D_{n}$ for infinitely many $n$; if $C \in \operatorname{cl}\left(\mathscr{D}_{X}\right)$, then either $C_{n} \subset D_{n}$ for infinitely many $n$ or $D_{n} \subset C_{n}$ for infinitely many $n$. In both cases we have either $C \subset D$ or $D \subset C$.

Proposition 5.7. If $X$ is a homogeneous, hereditarily unicoherent continuum, then $\mathscr{D}_{X}$ is closed in $C(X)$.

Proof. Let $D \in \operatorname{cl}\left(\mathscr{D}_{X}\right)$. According to Lemma 5.6, it suffices to show that $D$ is indecomposable. In the case $D \in K(X)$ it is indecomposable by Propositions 2.4 and 5.1. So suppose $D \notin K(X)$. Let $D=\operatorname{Lim}_{n} D_{n}, D_{n} \in \mathscr{D}_{X}$, and take a point $p \in D$. We can assume as in the proof of Lemma 5.6 that $p \in D_{n}$. If $h \in H(X)$ satisfies $h(D) \cap D \neq \varnothing$, then putting $C=h(D) \in \operatorname{cl}\left(\mathscr{D}_{X}\right)$ in Lemma 5.6 we obtain $h(D) \subset D$ or $D \subset h(D)$. Hence, by Proposition 2.3, for each $\delta$ there is a $\delta$-homeomorphism $h \in H(X)$ such that $h(D) \varsubsetneqq D$. Since $h(p) \in h\left(D_{n}\right) \cap D$ and $h\left(D_{n}\right) \in \mathscr{D}_{X}$, it follows from Lemma 5.6 that $h\left(D_{n}\right) \subset D$ or $D \subset h\left(D_{n}\right)$. But the last inclusion cannot occur for infinitely many $n$, otherwise $D \subset h(D) \varsubsetneqq D$. Being approximated by indecomposable subcontinua $h\left(D_{n}\right)$ (for $\delta \rightarrow 0$ ), the continuum $D$ is indecomposable in view of Proposition 2.1.

Now we come back to the class $\mathscr{F}$ of continua considered in $\S 4$. Homogeneous members of $\mathscr{F}$ contain no arc (Theorem 4.3). Combining this fact with Theorem 5.2, we get the following proposition.

Proposition 5.8. A homogeneous, hereditarily unicoherent continuum $X \in \mathscr{F}$ contains arbitrarily small, nondegenerate, indecomposable subcontinua.

Proposition 5.9. If $X \in \mathscr{F}$ is homogeneous and hereditarily unicoherent, then for each nondegenerate $K \in C(X)$ and each $p \in K$ there exists a maximal indecomposable, nondegenerate subcontinuum $C \subset K$ containing $p$.

Proof. It follows from the homogeneity of $X$ and Theorem 4.2 that there is an $A \in C(X)$ such that $p \in F(A), F(A)$ is nondegenerate, and $K-A \neq \varnothing$. Then $F(A) \subset K$. In view of Proposition 5.8, there is a small, indecomposable, nondegenerate subcontinuum $L$ of $X$ such that $p \in L$ and $F(A)-L \neq \varnothing$. Then $L \subset F(A)$ by Proposition 5.3. Thus $K$ has nondegenerate, indecomposable subcontinua containing $p$. The maximal one exists by Proposition 2.1.

The following proposition follows directly from Propositions 5.9 and 5.5.

Proposition 5.10. If $X \in \mathscr{F}$ is homogeneous and hereditarily unicoherent, then $\mathscr{D}_{X}$ contains arbitrarily small, nondegenerate continua. 
Denote by $\mathscr{D}_{X}(A)$ the family of all elements of $\mathscr{D}_{X}$ that are maximal with respect to the property of being contained in $A \in C(X)$.

Proposition 5.11. Let $X \in \mathscr{F}$ be homogeneous and hereditarily unicoherent and $A$ be a proper subcontinuum of $X$ with nondegenerate $F(A)$. Then $\mathscr{D}_{X}(A)$ forms a decomposition of $A$ into nondegenerate subcontinua which is continuous at each element $D \subset A-F(A)$. Moreover, $D$ is a terminal subcontinuum of $X$.

Proof. It is clear that $\mathscr{D}_{X}(A)$ forms a decomposition of $A$ by the definition of $\mathscr{D}_{X}$.

For each $p \in A$ there is a nondegenerate element $B \in \mathscr{D}_{X}$ with $p \in B$ and $\operatorname{diam} B<\operatorname{diam} F(A)$ by Proposition 5.10 and the homogeneity of $X$. Clearly, $B \subset A$, and thus all elements of $\mathscr{D}_{X}(A)$ are nondegenerate.

Let $D \in \mathscr{D}_{X}(A)$ be contained in $A-F(A)$. To show that $\mathscr{D}_{X}(A)$ is continuous at $D$, let a sequence of points $x_{n} \in A-F(A)$ converge to a point $x_{0} \in D$. Denote by $D_{n}$ the element of $\mathscr{D}_{X}(A)$ containing $x_{n}$. By Effros' Theorem 2.2, there are $\varepsilon_{n}$-homeomorphisms $h_{n} \in H(X)$ with $h_{n}\left(x_{n}\right)=x_{0}$ and $\lim \varepsilon_{n}=0$. We claim that $h_{n}\left(D_{n}\right)=D$ for sufficiently great $n$. In fact, if not, then applying Proposition 5.3 we have either $D \varsubsetneqq h_{n}\left(D_{n}\right)$ and $h_{n}\left(D_{n}\right) \subset A-F(A)$ or $D_{n} \varsubsetneqq h_{n}^{-1}(D)$ and $h_{n}^{-1}(D) \subset A-F(A)$, a contradiction to the definition of $\mathscr{D}_{X}(A)$. Hence, the decomposition is continuous at $D$.

Suppose now that some $D \in \mathscr{D}_{X}(A)$ which is contained in $A-F(A)$ is not terminal; that is, there is a $C \in C(X)$ satisfying

$$
C \cap D \neq \varnothing \quad \text { and } \quad C-D \neq \varnothing \neq D-C .
$$

Since the decomposition $\mathscr{D}_{X}(A)$ is continuus at $D$, one can enlarge the continum $C \cap D$ a little bit to obtain a continuum $C_{1}$ satisfying $(*)$ such that $C \cap D \subset C_{1} \subset C, C_{1}$ contains no element of $\mathscr{D}_{X}(A)$, and $C_{1} \subset A-F(A)$. Then $C_{1}$ contains a continuum $I$ irreducible with respect to the property that it intersects $D$ and some fixed $E \in \mathscr{D}_{X}(A), E \neq D$. Certainly, $I$ also intersects some $P \in \mathscr{D}_{X}(A), D \neq P \neq E$. Now, using an idea from the proof of Theorem 5.2, we start to construct an indecomposable continuum $K \subset X$ such that

$$
P-K \neq \varnothing \neq K-P, \quad P \cap K \neq \varnothing,
$$

obtaining a contradiction because $P \in \mathscr{D}_{X}$.

Choose a point $p \in P-I$ and put $\alpha=d(p, I \cup D \cup E), \beta=\inf \{d(x, y): x \in$ $I \cup D \cup E, y \in F(A)\}, \varepsilon=\min \{\alpha, \beta\} / 2$. By the Effros Theorem 2.2 we find an $\varepsilon / 4$-homeomorphism $h_{1} \in H(X)$ such that $h_{1}(D \cap I)$ intersects a composant of $D$ different from that containing the continuum $D \cap I, D-h_{1}(I) \neq \varnothing$, and $h_{1}(E) \cap D=\varnothing$. Observe that $h_{1}(I \cup E) \cap(I \cup E)=\varnothing$ by the hereditary unicoherence of $X$. The union $I \cup D \cup h_{1}(I) \subset A-F(A)$ contains a subcontinuum $I_{1}$ irreducible with respect to the property that it intersects $E_{1}=E$ and $D_{1}=h_{1}(E) ; I_{1}$ satisfies $D_{1}-I_{1} \neq \varnothing \neq E_{1}-I_{1}$. Next, find 
an $\varepsilon$ /8-homeomorphism $h_{2}: X \rightarrow X$ such that $h_{2}\left(D_{1} \cap I_{1}\right)$ intersects a composant of $D_{1}$ different from that containing $D_{1} \cap I_{1}, D_{1}-h_{2}\left(I_{1}\right) \neq \varnothing$, and $h_{2}\left(E_{1}\right) \cap D_{1}=\varnothing$, etc. Continuing this construction, an increasing sequence of irreducible continua $I_{n}$ is obtained such that $K=\operatorname{Lim}_{n} I_{n} \subset A-F(A)$. The continuum $K$ is indecomposable, by an argument similar to that from the proof of Theorem 5.2, and $K$ satisfies $(* *)$.

The proof of Proposition 5.11 is complete.

Theorem 5.12. If $X \in \mathscr{F}$ is homogeneous and hereditarily unicoherent, then $X$ contains arbitrarily small, nondegenerate, terminal subcontinua.

Proof. $X$ contains arbitrarily small continua $A$ with nondegenerate $F(A)$ by Theorem 4.2.

If $A=F(A)$, then $A$ is terminal. If $A-F(A) \neq \varnothing$, then an element of $\mathscr{D}_{X}(A)$ contained in $A-F(A)$ is a nondegenerate, terminal subcontinuum of $X$ (Proposition 5.11).

\section{TREELIKE, HOMOGENEOUS CONTINUA}

Let us recall that a point $x$ of a continuum $X$ is said to be an end point of $X$ if and only if for each $K, L \in C(X)$ if $x \in K \cap L$, then either $K \subset L$ or $L \subset K$ [1, (B), p. 660].

It is clear that a continuum $X$ is hereditarily indecomposable if and only if each point of $X$ is an end point.

Let $X$ be a continuum. We will consider the following condition:

(t) for each nondegenerate continuum $T \in \mathscr{T}_{X}$ there exists a sequence $\left\{T_{n}\right\} \subset \mathscr{T}_{X}, n=1,2, \ldots$, such that $T_{n} \varsubsetneqq T$ and $\operatorname{Lim}_{n} T_{n}=T$.

Obviously, hereditarily indecomposable, nondegenerate continua $X$ have property $(\mathrm{t})$, since $C(X)=\mathscr{T}_{X}$.

Proposition 6.1. If a continuum $X$ with property $K$ satisfies condition $(\mathbf{t})$, then $\mathscr{T}_{X}$ contains an order arc from a singleton $\{x\}$ to $X$ and $x$ is an end point of $X$.

Proof. Observe first that

(i) for each $T \in \mathscr{T}_{X}$ the set $C(T) \cap \mathscr{T}_{X}$ is connected.

In fact, suppose $C(T) \cap \mathscr{T}_{X}=P \cup Q$ with $P, Q$ being closed and disjoint. Since the set $\{\{x\}: x \in T\} \subset C(T) \cap \mathscr{T}_{X}$ is connected, it is contained in one of these sets, say in $P$. If $Q$ were nonempty, it would contain nondegenerate, minimal elements, contrary to $(\mathrm{t})$.

Let $\mu: C(X) \rightarrow[0,1]$ be a Whitney map with $\mu(X)=1$. Then, by (i), we can find a collection $\mathscr{W}_{n}=\left\{W_{n}^{n}, W_{n-1}^{n}, \ldots, W_{0}^{n}\right\} \subset \mathscr{T}_{X}$ such that $W_{i}^{n} \subset W_{i+1}^{n}$ and $\mu\left(W_{i}^{n}\right)=i / n$ for $n=1,2, \ldots$. The sequence $\left\{\mathscr{W}_{n}\right\}, n=1,2, \ldots$, has a subsequence converging to some family $\mathscr{W} \subset C(X)$. Observe that $\mathscr{W} \subset \mathscr{T}_{X}$ by Proposition 2.9 and that $\mathscr{W}$ is an order arc from a singleton $\{x\}$ to $X$. 
Finally, we will show that the point $x$ is an end point of $X$. Indeed, suppose $K, L \in C(X), x \in K \cap L$, and $K-L \neq \varnothing \neq L-K$. Then there are a number $s$ such that $\max \{\mu(K), \mu(L)\}<s<\mu(K \cup L)$ and a $T \in \mathscr{W}$ with $\mu(T)=s$. We have $K \subset T, L \subset T$, and $K \cup L \nsubseteq T$, a contradiction.

Since confluent mappings preserve the property of being an end point of a continuum, the next fact follows from Proposition 6.1.

Proposition 6.2. A nondegenerate continuum $X$ with property $K$ which is homogeneous with respect to confluent mappings satisfies $(\mathrm{t})$ if and only if $X$ is hereditarily indecomposable.

Lemma 6.3. Let $X$ be a homogeneous, nondegenerate continuum such that

(1) $X$ is treelike or

(2) each proper subcontinuum of $X$ is treelike and $\mathscr{T}_{X}-\{X\}$ is not compact. Then $X$ satisfies condition $(\mathrm{t})$.

Proof. If not, then there is a nondegenerate continuum $T \in \mathscr{T}_{X} \cap K(X)$ in case (1) $\left(T \in\left(\mathscr{T}_{X} \cap K(X)\right)-\{X\}\right.$ in case (2)) by Proposition 2.3. So $T$ is a homogeneous, treelike continuum (Proposition 2.4).

Let $\mathscr{T}_{X}(T)$ be the family of all maximal terminal, proper subcontinua of $T$. Clearly, $\mathscr{T}_{X}(T)$ is a partition of $T$. Since $h(P) \in \mathscr{T}_{X}(T)$ for each $P \in \mathscr{T}_{X}(T)$ and each $h \in H(T)$, the family $\mathscr{T}_{X}(T)$ is a continuous decomposition of $T$ with the quotient space $T / \mathscr{T}_{X}(T)$ being a nondegenerate, treelike, homogeneous continuum which contains no proper, terminal subcontinuum (see [23, Theorem 1, p. 138]). But nondegenerate, treelike, homogeneous continua are in class $\mathscr{F}$ (Theorem 3.4), therefore $T / \mathscr{T}_{X}(T)$ contains a nondegenerate, proper, terminal subcontinuum (Theorem 5.12), a contradiction.

Our main result now follows from Lemma 6.3 and Proposition 6.2.

Theorem 6.4. Each homogeneous, treelike continuum is hereditarily indecomposable.

The next theorem is a combination of the above investigations with two results of J. T. Rogers, Jr.

Theorem 6.5. Let $X$ be a nondegenerate, homogeneous continuum. Then the following conditions are equivalent:

(1) $X$ is treelike;

(2) $X$ is hereditarily indecomposable;

(3) $X$ is one-dimensional and the set $\mathscr{T}_{X}-\{X\}$ is not compact;

(4) $X$ is one-dimensional and acyclic.

Proof. (1) $\Rightarrow(2)$ is established in Theorem 6.4. (2) $\Rightarrow(1)$ is proved in [22]. Obviously, conditions (1) and (2) together imply (3).

To show $(3) \Rightarrow(2)$ assume there is a sequence $\left\{T_{n}\right\} \subset \mathscr{T}_{X}-\{X\}$ converging to $X$. By Effros' Theorem 2.2, we can assume $p \in T_{n}$ for some fixed point $p \in$ $X$ and for each $n=1,2, \ldots$. Since $T_{n}$ are terminal, for each $C \in C(X)-\{X\}$ with $p \in C$ and for some $n$, we have $C \subset T_{n}$. Further, each $T_{n}$ is treelike by 
[16, Corollary 1.13] and so $C$ is treelike. Therefore each proper subcontinuum of $X$ is treelike, since $X$ is homogeneous. Finally, we obtain (2) by Lemma 6.3 and Proposition 6.2. Hence, conditions (1)-(3) are equivalent.

The implication $(4) \Rightarrow(1)$.is proved in [28, Corollary 6.5], and its converse is well known. The proof is complete.

Now we can easily obtain a general decomposition theorem for homogeneous curves (i.e., one-dimensional continua).

Theorem 6.6. If $X$ is a homogeneous curve, then there is the unique continuous decomposition $\mathscr{D}$ of $X$ such that

(a) each $D \in \mathscr{D}$ is a hereditarily indecomposable treelike, homogeneous continuum which is terminal in $X$,

(b) $h(D) \in \mathscr{D}$ for each $D \in \mathscr{D}$ and $h \in H(X)$, and

(c) $X / \mathscr{D}$ is a homogeneous curve with no proper, nondegenerate, terminal subcontinua.

Proof. If the set $\mathscr{T}_{X}-\{X\}$ is not compact, then we put $\mathscr{D}=\{X\}$ and the conclusion holds by Theorem 6.5. Otherwise, let $\mathscr{D}$ be the collection of all maximal elements of $\mathscr{T}_{X}-\{X\}$. Then it is clear that condition (b) holds. Hence, the conclusion follows from [24, Theorem 4, p. 377] and Theorem 6.4, because cell-like curves are treelike.

Remark 1. A conclusion similar to that of Theorem 6.6 has been obtained by Rogers [27] provided $H^{1}(X) \neq 0$ (Rogers has not assumed that $\operatorname{dim} X=1$ ). The methods of [27] are very different from those of this paper.

Corollary 6.7. Each proper, temrinal subcontinuum $T$ of a homogeneous curve $X$ is hereditarily indecomposable and treelike. In other words, each subcontinuum of $T$ is terminal in $X$.

We will use Theorem 6.6 to get a short proof of Hagopian's classification of homogeneous, atriodic continua. Originally it was presented in [8].

Recall firstly the following result [17, (13.3), p. 31 and (6.13), p. 18].

Proposition 6.8. If $X$ is a homogeneous, atriodic continuum, then $Q \in C(X)-$ $\{X\}$ is indecomposable if and only if $Q$ is terminal.

Theorem 6.9 [8]. Let $X$ be a homogeneous, atriodic continuum. Then there is a unique continuous decomposition $\mathscr{D}$ of $X$ such that:

(a) each element of $\mathscr{D}$ is a homogeneous, hereditarily indecomposable, treelike continuum which is terminal in $X$,

(b) $h(D) \in \mathscr{D}$ for each $D \in \mathscr{D}$ and $h \in H(X)$, and

(c) $X / \mathscr{D}$ is either a point or a solenoid.

Proof. Atriodic, homogenous continua are known to be one-dimensional. Originally it was proved in [8], but a quick proof of this fact has been found in [26] and in [16, Corollary 3.5]. Thus, consider the decomposition $\mathscr{D}$ of $X$ from 
Theorem 6.6. The continuum $Y=X / \mathscr{D}$ is atriodic, homogeneous, and contains no proper, nondegenerate, terminal subcontinua. According to Proposition 6.8 , if $Y$ is nondegenerate, it contains a nondegenerate, hereditarily decomposable continuum. To complete the proof apply the result of $[17,(14.8)$, p. 35] saying that atriodic, homogeneous continua with nondegenerate, hereditarily decomposable subcontinua are solenoids.

Remark 2. It is remarkable that, in the case of an atriodic, treelike, homogeneous continuum $X$, Proposition 6.8 allows us to simplify the proof that $X$ is hereditarily indecomposable. Namely, $X$ contains no arc (see, e.g., [23], [7], [13], or our Corollary 4.5), so by Theorem 5.2, $X$ contains nondegenerate, proper, terminal subcontinua and all results of $\S 6$ are valid for $X$. In such a way we omit Propositions 5.3-5.12 (and $\S \S 3,4$ if we use, say, [23]).

\section{FinAl REMARKS AND PROBLEMS}

Problem 7.1. Suppose a homogeneous continuum $X$ contains a nondegenerate, proper, terminal subcontinuum $Y$. Does it follow that $\operatorname{dim} X=1$ ? (If $\operatorname{dim} Y=1$, then the answer is yes.)

Problem 7.2. Assume $X$ is a homogeneous continuum such that each indecomposable subcontinuum of $X$ is terminal (then $\operatorname{dim} X \leq 1[16,(3.6)]$ ). Must $X$ be atriodic?

Problem 7.3. Let $X$ be a homogeneous curve with no proper, nondegenerate, terminal subcontinua. Must $X$ contain an arc? Is $X$ a solenoid in the case that $X$ is indecomposable (compare [25, Question 6, p. 221])?

Problem 7.4. What is a relationship between homogeneous, indecomposable curves and homogeneous, hereditarily unicoherent continua?

The authors wonder how far one can extend Theorems 5.2 and 6.4 for some (larger than $H(X))$ classes of mappings. The results of $\S \S 3,4$ and Proposition 6.2 suggest possible further investigations of treelike continua which are homogeneous with respect to confluent (open) light mappings.

We are also encouraged by recent successful generalizations of some important theorems on homogeneous continua. Let us mention two of them.

1. If a continuum $X$ is homogeneous with respect to open mappings and each subcontinuum of $X$ is an arc, then $X$ is a solenoid [20].

2. If a proper subcontinuum $X$ of a 2-manifold is homogeneous with respect to open light mappings and $X$ contains an arc, then $X$ is a simple closed curve [21].

One can see that some versions of Effros' theorem which are formulated in [4, Proposition 3.1, Theorem 5.9] provide fundamental tools for studying generalized homogeneity of continua. 
Assume a treelike continuum (or hereditarily unicoherent continuum with no arcs) $X$ is homogeneous with respect to confluent (confluent light, monotone, open, open light, monotone open) mappings. The first question in the subsequent group of problems is the most general.

Problem 7.5. Is $X$ is hereditarily indecomposable?

Problem 7.6. Does $X$ contain proper, nondegenerate, terminal subcontinua?

Problem 7.7. Does $X$ contain proper, nondegenerate, indecomposable subcontinua?

Problem 7.8. Is $X$ indecomposable?

\section{REFERENCES}

1. R. H. Bing, Snake-like continua, Duke Math. J. 18 (1951), 653-663.

2. _ A simple closed curve is the only homogeneous bounded plane continuum that contains an arc, Canad. J. Math. 12 (1960), 209-230.

3. J. J. Charatonik, The property of Kelley and confluent mappings, Bull. Acad. Polon. Sci. Sér. Sci. Math. 31 (1983), 375-380.

4. J. J. Charaonik and T. Maćkowiak, Around Effros' theorem, Trans. Amer. Math. Soc. 298 (1986), 579-602.

5. E. G. Effros, Transformation groups and $C^{*}$-algebras, Ann. of Math. (2) 81 (1965), 38-55.

6. C. L. Hagopian, Indecomposable homogeneous plane continua are hereditarily indecomposable, Trans. Amer. Math. Soc. 224 (1976), 339-350.

7. __ No homogeneous tree-like continuum contains an arc, Proc. Amer. Math. Soc. 88 (1983), 560-564.

8. __ Atriodic homogeneous continua, Pacific J. Math. 113 (1984), 333-347.

9. F. B. Jones, Certain homogeneous unicoherent indecomposable continua, Proc. Amer. Math. Soc. 2 (1951), 855-859.

10. _ Homogeeous plane continua, Proc. Auburn Topology Conference, Auburn University, 1969, 45-56.

11. H. Kato, Generalized homogeneity of continua and a question of J. J. Charatonik, Houston J. Math. 13 (1987), 51-63.

12. J. L. Kelley, Hyperspaces of a continuum, Trans. Amer. Math. Soc. 52 (1942), 22-36.

13. P. Krupski, On homogeneous tree-like continua, Rend. Circ. Mat. Palermo (2), Suppl. 18 (1988), 327-336.

14. K. Kuratowski, Topology, vol. I, Academic Press and PWN, New York, London, Warsaw, 1966.

15. __ Topology, vol. II, Academic Press and PWN, New York, London, Warsaw, 1968.

16. T. Maćkowiak, Terminal continua and the homogeneity, Fund. Math. 127 (1987), 177-186.

17. T. Maćkowiak and E. D. Tymchatyn, Continuous mappings on continua II, Dissertationes Math. (Rozprawy Mat.) 225 (1984), 1-57.

18. S. B. Nadler, Jr., Hyperspaces of sets, Marcel Dekker, New York, 1978.

19. J. R. Prajs, Homogeneous, tree-like continua are hereditarily indecomposable, preprint.

20. __ Openly homogeneous continua having only arcs for proper subcontinua, Topology Appl. 31 (1989), 133-147.

21. __ Openly homogeneous continua in 2-manifolds, preprint. 
22. J. T. Rogers, Jr., Homogenneous, hereditarily indecomposable continua are tree-like, Houston J. Math. 8 (1982), 421-428.

23. __ Decompositions of homogeneous continua, Pacific J. Math. 99 (1982), 137-144.

24. __ Cell-like decompositions of homogeneous continua, Proc. Amer. Math. Soc. 87 (1983), 375-377.

25. __ Homogeneous continua, Topology Proc. 8 (1983), 213-233.

26. __ Atriodic homogeneous nondegenerate continua are one-dimensional, Proc. Amer. Math. Soc. (to appear).

27. __ Decompositions of continua over the hyperbolic plane, Trans. Amer. Math. Soc. (to appear).

28. __ Hyperbolic ends and continua, preprint.

29. G. S. Ungar, On all kinds of homogeneous spaces, Trans. Amer. Math. Soc. 212 (1975), 393400.

30. G. T. Whyburn, Analytic topology, Amer. Math. Soc. Colloq. Publ., vol. 28, Amer. Math. Soc., Providence, R. I., 1963.

Mathematical Institute, University of Wroclaw, 50-384 Wroclaw, Poland

Institute of Mathematics, Opole Pedagogical University, 45-951 Opole, Poland 